\title{
BMJ Open Community-based physical activity and nutrition programme for adults with metabolic syndrome in Vietnam: study protocol for a cluster-randomised controlled trial
}

\author{
Van Dinh Tran, ${ }^{1,2}$ Andy H Lee, ${ }^{2}$ Jonine Jancey, ${ }^{2,3}$ Anthony $\mathrm{P}$ James, ${ }^{2}$ \\ Peter Howat, ${ }^{2,3}$ Le Thi Phuong Mai ${ }^{1}$
}

To cite: Tran VD, Lee AH, Jancey J, et al. Communitybased physical activity and nutrition programme for adults with metabolic syndrome in Vietnam: study protocol for a clusterrandomised controlled trial. BMJ Open 2016;6:e011532. doi:10.1136/bmjopen-2016011532

- Prepublication history and additional material is available. To view please visit the journal (http://dx.doi.org/ 10.1136/bmjopen-2016011532).

Received 16 February 2016 Revised 14 May 2016 Accepted 17 May 2016

\section{CrossMark}

\footnotetext{
${ }^{1}$ Department of Community Health and Network Coordination, National Institute of Hygiene and Epidemiology, Hanoi, Vietnam

${ }^{2}$ School of Public Health, Curtin University, Perth, Western Australia, Australia ${ }^{3}$ Collaboration for Evidence, Research and Impact in Public Health, Curtin University, Perth, Western Australia, Australia
}

Correspondence to Van Dinh Tran; tranvandinhnihe@gmail.com

\begin{abstract}
Introduction: Metabolic syndrome (MetS) is a cluster of risk factors for cardiovascular diseases and type II diabetes. In Vietnam, more than one-quarter of its population aged 50-65 have MetS. This clusterrandomised controlled trial aims to evaluate the effectiveness of interventions to increase levels of physical activity and improve dietary behaviours among Vietnamese adults aged $50-65$ years with MetS.
\end{abstract}

Method and analysis: This 6-month communitybased intervention includes a range of strategies to improve physical activity and nutrition for adults with MetS in Hanam, a province located in northern Vietnam. 600 participants will be recruited from 6 communes with 100 participants per commune. The 6 selected communes will be randomly allocated to either an intervention group $(m=3 ; n=300)$ or a control group $(m=3 ; n=300)$. The intervention comprises booklets, education sessions, resistance bands and attending local walking groups that provide information and encourage participants to improve their physical activity and healthy eating behaviours during the 6month period. The control group participants will receive standard and 1-time advice. Social cognitive theory is the theoretical concept underpinning this study. Measurements will be taken at baseline and postintervention to evaluate programme effectiveness. Ethics and dissemination: The research protocol was approved by the Curtin University Human Research Ethics Committee (approval number: HR139/ 2014). The results of the study will be disseminated through publications, reports and conference presentations.

Trial registration number: ACTRN12614000811606.

\section{INTRODUCTION}

Vietnam is a developing country with a population of more than 90 million. ${ }^{1}$ With socioeconomic development occurring rapidly

\section{Strengths and limitations of this study}

- This is the first community-based randomised controlled trial undertaken on Vietnamese adults with MetS. Finding will be potentially applicable for non-communicable disease prevention in Vietnam.

- Intervention strategies are developed taking into account the cultural context and underpinned by theory.

- Challenges in participant recruitment and minimising attrition during the follow-up of the intervention.

- Long-term follow-up to assess sustainability of the programme is not feasible due to budget constraints.

during the past two decades, risk factors for non-communicable diseases (NCDs) such as hypertension, overweight/obesity and physical inactivity are becoming increasingly prevalent. $^{2}$ NCDs are rising rapidly, accounting for $71 \%$ of the total disease burden in Vietnam. Cardiovascular events alone are responsible for about 110000 deaths each year or $20 \%$ of the total mortality, while the prevalence of type II diabetes (T2D) has tripled in the past two decades and continues to rise. ${ }^{3}$

Metabolic syndrome (MetS) is a cluster of risk factors for cardiovascular diseases (CVDs) and T2D, including abdominal obesity, elevated blood pressure, reduced high-density lipoprotein cholesterol (HDL-C), elevated triglyceride levels and raised fasting plasma glucose. ${ }^{4}$ People with MetS have twice the risk of suffering CVD over the next 5-10 years compared with those without the condition, together with another fivefold increase in risk of incurring T2D. ${ }^{45}$ 
Several definitions of MetS have been proposed by different organisations over the past decade, but the most common criteria ${ }^{6}$ came from the WHO ${ }^{7}$ the National Cholesterol Education Programme Adult Treatment Panel III (ATP III) ${ }^{8}$ and the International Diabetes Federation (IDF). ${ }^{5}$ These definitions exhibit common features, but their differences in several parameters have led to challenges in terms of applicability and establishing positive predictive values. ${ }^{6}$ Among them, waist circumference in defining abdominal obesity in different populations and ethnicities is the most controversial parameter. Consequently, the IDF has proposed a new set of waist circumference criteria with ethnic/racial specific cut-offs that are applicable across populations. ${ }^{4}$

MetS is now a global concern, ${ }^{4}$ with an increasing trend worldwide, yet it is often undiagnosed. ${ }^{9} 10$ The prevalence of MetS varies, ranging from $<10 \%$ to $84 \%$, depending on the region, sex, age and ethnicity of the population being studied. ${ }^{11}$ Globally, almost one-quarter of the adult population have MetS. ${ }^{5}$ In Vietnam, MetS is a major public health problem, with a high prevalence particularly in urban areas. ${ }^{12-14}$ It is estimated that $18 \%$ of Vietnamese adults aged 35-65 years have MetS whose rate increases with age. ${ }^{15} \mathrm{~A}$ recent study conducted in northern Vietnam indicated that the prevalence of MetS among those in the age group 55-64 years is $~ 27 \% .^{16}$ The study also suggested that $40 \%$ of Vietnamese aged 40-64 years possess at least two of the five components of MetS, the so-called 'pre-MetS'. ${ }^{16}$ Modifiable lifestyle factors, such as inadequate levels of physical activity and poor dietary habits, are known to be associated with the development of MetS. ${ }^{13} 15$

Weight reduction through behavioural changes, such as reducing energy intake and increasing physical activity levels, thereby enhancing energy expenditure, are the first priorities of MetS management. ${ }^{17}{ }^{18}$ Previous studies have demonstrated the effectiveness of lifestyle interventions aimed at increasing physical activity and achieving weight reduction, while reducing the prevalence of MetS and its components. ${ }^{19-22}$ For example, the Dietary Approaches to Stop Hypertension diet could reduce blood pressure ${ }^{23}$ and the risk of $\mathrm{CVD},{ }^{24}$ while the Diabetes Prevention Program, a lifestyle intervention to control weight and increase activity levels, was successful in delaying the development of T2D. ${ }^{25}$ Similarly, the Strong Women-Healthy Hearts Program was a community-based intervention to reduce the risk of CVD in sedentary midlife women through fitness and nutrition. ${ }^{26}$ Despite such significant evidence emerging, and other studies from developed countries, ${ }^{27} 28$ there is still a lack of intervention research in developing countries with emerging economies. ${ }^{16}$

In view of the high prevalence of MetS and pre-MetS in Vietnam, appropriate interventions and healthy lifestyle programmes must be developed and implemented in a timely manner. ${ }^{12}{ }^{16}$ This paper describes the protocol of a cluster-randomised controlled trial (RCT) for community-dwelling adults aged 50-65 years with MetS in Vietnam. It is hypothesised that after the 6-month intervention, levels of physical activity, dietary behaviours, lipid profile and fasting glucose, as well as anthropometric measures among the intervention group, will demonstrate statistically significant improvements when compared with the control group.

\section{METHODS AND ANALYSIS}

\section{Study design}

This 6-month community-based cluster RCT is designed to improve physical activity and dietary behaviours for adults aged 50-65 years with MetS in Hanam province located in northern Vietnam. Hanam province comprises five districts and one city, with a total population of 799400 in $2014 .^{1}$ It is estimated that $\sim 950$ people aged 50-65 years reside within each of the 98 communes in the province. Given this number of target residents per commune, and a conservative estimate of MetS prevalence $(11 \%)$, six communes $(\mathrm{N}=5700)$ will be selected using random numbers and then assigned randomly to either the intervention group $(\mathrm{m}=3)$ or the control group $(m=3)$, with about 100 eligible participants with MetS recruited per commune (cluster). Data will be collected at baseline and postintervention (6 months later) to assess changes in outcome measures. Figure 1 shows the schematic diagram of the study timeline.

\section{Power calculation}

The power calculations are based on a logistic mixed regression model of the binary outcome variable moderate activity participation (yes, no) for each participant. We hypothesise that after the 6-month intervention, the intervention group will demonstrate an increase of $10 \%$ in moderate activity prevalence when compared with the control group. A final sample size of $n=480$ will provide sufficient power $(80 \%)$ to detect such a medium effect size at $5 \%$ significance level for the group by time interaction term in the logistic mixed regression model accounting for gender but without other covariate adjustment. To allow for $80 \%$ complete data across the two assessments due to attrition and withdrawals, a total of $n=600$ participants with MetS will be initially recruited from the six selected communes at baseline. The power calculations were performed using the STATA software Release V.13 (StataCorp. Stata: Release 13. Statistical Software. College Station, Texas: StataCorp LP. 2013).

\section{Recruitment of participants}

\section{Screening}

The RCT targets adults aged 50-65 years with MetS residing in six communes in Hanam province. The selected communes should be similar in terms of socioeconomic

\begin{tabular}{|l|c|c|c|}
\hline Study group & 0 month (Baseline) & Intervention & 6 month (Post-test) \\
\hline Intervention $(\mathrm{n}=300)$ & $\mathrm{O} 1$ & $\mathrm{X}$ & $\mathrm{O} 2$ \\
\hline Control $(\mathrm{n}=300)$ & $\mathrm{O} 1$ & & $\mathrm{O} 2$ \\
\hline
\end{tabular}
$\mathrm{O}=$ Observation $\quad \mathrm{X}=$ Intervention

Figure 1 Study design. 
and geographic conditions. A list of adults aged 5065 years currently living in these communes will be obtained from the Hanam Provincial Preventive Medicine Centre. Potential participants $(\mathrm{N}=5700)$ will be invited to attend their local commune health centre to have their height and weight measured. Small incentives will be provided to encourage attendance. A screening interview seeking basic information on age, gender and physical activity levels will be administered face to face using a brief questionnaire. Body mass index will be classified using the cut-off points for Asian populations recommended by the WHO: $<18.5$, underweight; 18.5 to $<23$, normal weight; 23-27.5, overweight; $>27.5$, obese. ${ }^{29}$ In terms of exclusion criteria, individuals who have been receiving treatment for T2D, CVD, hypertension, raised lipid profile, raised glucose, or have taken part in any dietary and/or physical activity programme within the past year will be excluded ( $\approx 10 \%$ of the target group).

\section{Determining MetS status}

With almost $37 \%$ of Vietnamese adults aged $50-60$ years being overweight ${ }^{30}$ and $31.9 \%$ of them having MetS, ${ }^{16}$ approximately $\mathrm{N}=1900$ will be classified as overweight/ obese and subsequently invited to undertake a blood test and have their waist circumference, hip circumference and blood pressure measured to confirm MetS status $(n=600)$.

A single overnight fasting blood sample will be collected and analysed to determine fasting plasma glucose, triglyceride, total cholesterol and HDL-C levels. On the basis of these results, people suspected to be diabetic (fasting plasma glucose $\geq 7.0 \mathrm{mmol} / \mathrm{L}$ or $>126 \mathrm{mg}$ / $\mathrm{dL}$ ) will be excluded from the study and recommended to attend a local hospital for confirmation of T2D instead. MetS status will be determined using the modified ATP III criteria with the waist circumference for Asia population being adopted, ${ }^{4}$ which includes any three of the following five risk factors (inclusion criteria): $:^{8}$

- Waist circumference (male $\geq 90 \mathrm{~cm}$, female $\geq 80 \mathrm{~cm}$ for Asia population; $\left.{ }^{4}\right)$

- Raised triglycerides $(\geq 1.7 \mathrm{mmol} / \mathrm{L}$ or $150 \mathrm{mg} / \mathrm{dL})$;

- Reduced HDL-C $(<1.03 \mathrm{mmol} / \mathrm{L}$ or $40 \mathrm{mg} / \mathrm{dL}$ for males, $<1.29 \mathrm{mmol} / \mathrm{L}$ or $50 \mathrm{mg} / \mathrm{dL}$ for females);

- Raised blood pressure (systolic $\geq 130 \mathrm{~mm} \mathrm{Hg}$ or diastolic $\geq 85 \mathrm{~mm} \mathrm{Hg}$ );

- Raised plasma glucose (fasting plasma glucose $\geq 6.1 \mathrm{mmol} / \mathrm{L}$ ).

\section{Allocation to control and intervention groups}

The six communes (intervention $\mathrm{m}=3$, controls $\mathrm{m}=3$ ) will be randomly allocated to either the intervention or the 'wait-listed' control group using a table of random numbers, which are geographically separated to avoid contamination and information exchange between groups, giving a total of 600 participants at baseline evaluation. A staff member from the Hanam Provincial
Preventive Medicine Centre will independently perform the cluster-randomisation process. Figure 2 summarises the CONSORT flow chart of the study procedure.

\section{Theoretical basis}

Social cognitive theory (SCT), which underpins this research, is built on an understanding that the interactions between individual characteristics and environmental events shape how a person behaves. ${ }^{31} 32$ The SCT perspective is that people are not driven solely by inner forces and not automatically shaped by external influences. Rather, human behaviour can be explained through its interaction with environmental events. These complex yet subtle interactions should be considered and well understood as they are essential to informing behavioural change interventions. ${ }^{33}$ A key construct of SCT is self-efficacy, which is concerned with an individual's belief in their ability to perform a specific task. Self-efficacy is behaviour specific and invaluable in achieving successful behaviour change, such as increasing levels of physical activity or improving dietary intake. ${ }^{33}$ Other major constructs of SCT are outcome expectations, reinforcement (motivation/incentives), observational learning, goal setting and the environment. All these constructs will help to inform the intervention strategies.

\section{Intervention}

Description

The adopted intervention strategies are based on our previous successful interventions, ${ }^{21}{ }^{34}$ as research has indicated that a combination of health education and walking programmes can lead to improved lifestyle management and reduced risk of MetS. ${ }^{35}$ This communitybased intervention will include a range of strategies and resources to encourage participation and to improve retention, such as education sessions, information booklet, resistance band and walking groups led by local walk leaders. All components of the programme will be conducted within the participant's commune to minimise participant burden. They are designed to inform participants of the benefits of being physically active and maintaining a healthy diet as a means of preventing chronic disease. Positive reinforcement, by way of encouragement and feedback, will be facilitated through the trained walking group leaders.

\section{Walk leaders}

A walk leader in each walking group will be either a local middle-aged volunteer or the village health worker within the commune. The walk leaders are required to be enthusiastic, demonstrate leadership qualities and be respected by the commune residents. The research team will maintain regular contact and monitor progress with them via telephone and a monthly face-to-face meeting. They are required to attend three 2-hour training sessions and will be provided with a package containing the 

the research procedure. BMI, body mass index; MetS, metabolic syndrome.
Figure 2 The flow diagram of

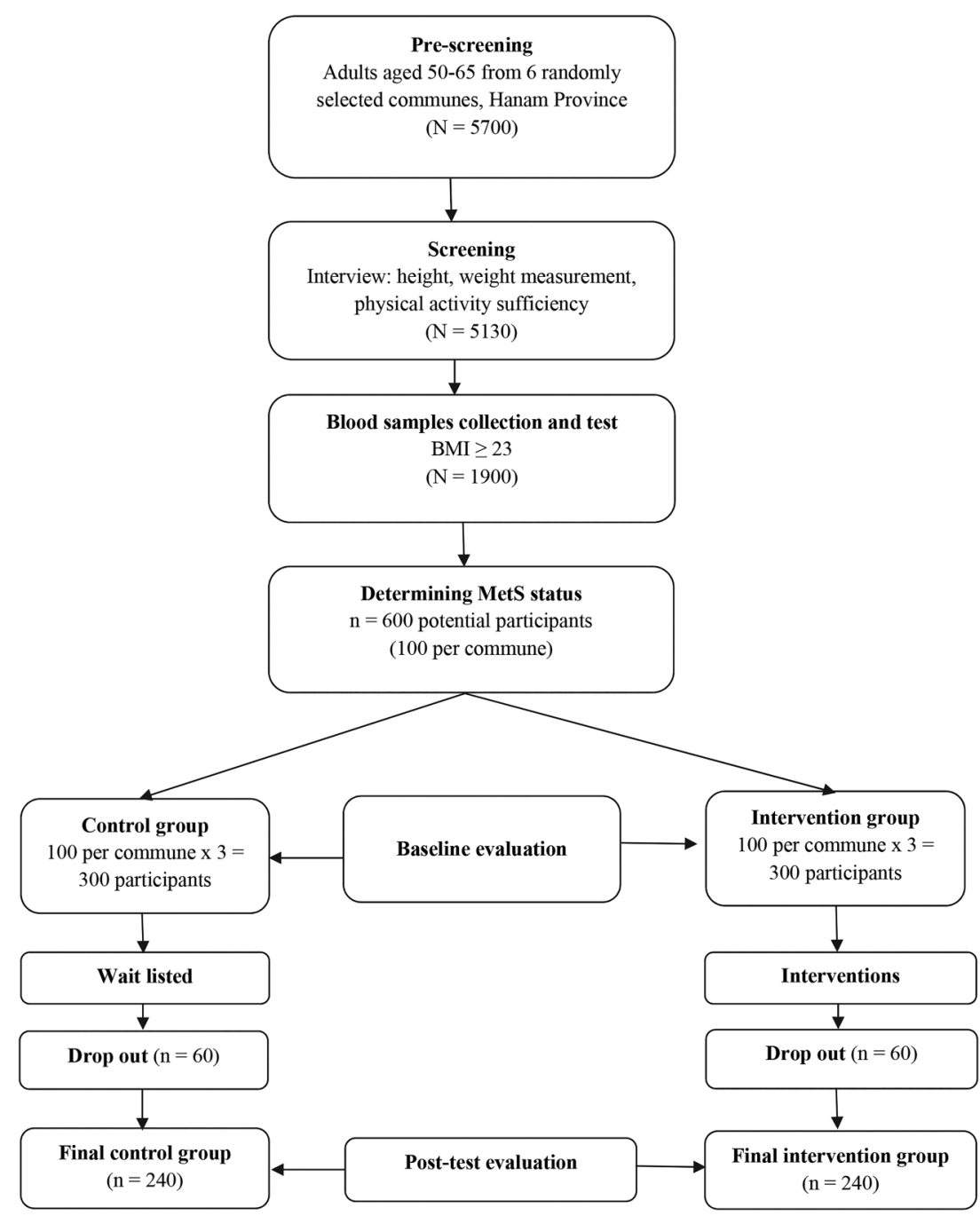

education materials, as well as a manual for managing the group walks. The manual is a comprehensive guide to mobilise walking groups, containing examples on walking activities and stretching and resistance exercises, benefits of being physically active and maintaining a healthy diet, supplemented by effective communication strategies. Besides mobilising and encouraging the participants for group walking, the group leaders also guide the participants but have the flexibility to tailor the prescribed programme to suit individual needs, such as walking less or more, within their group. They will also be responsible for administrative work such as recording participant contact details.

\section{Programme delivery}

Programme staff will be selected from medical doctors and nurses who are employed by the Hanam Provincial Preventive Medicine Centre. These staff members will be responsible for conducting interviews and taking anthropometric measurements and blood sample collections, after receiving intensive training from the chief investigators, who oversee all aspects of the programme delivery including the development of resources, setting up of walking groups and process evaluation.

\section{Programme resources}

\section{Education sessions}

The participants $(\mathrm{n}=100)$ in each intervention commune will be divided into four groups, that is, 25 participants per group. Each group will attend four 2-hour education sessions on healthy diet and physical activity in months 1, 2, 3 and 4 of the programme held at the local commune health centres. The duration and size of these education sessions are determined on the basis of a previous study. ${ }^{36}$ Sessions will be participatory and interactive, rather than didactic. Small group activities will foster problem-solving, social support and programme ownership. Table 1 describes the contents of the four education sessions and their linkages with behaviour change underpinned by the SCT.

\section{Information booklet}

At the first education session, each participant will be given a booklet (in Vietnamese) designed to inform and support improvements in nutritional intake and physical activity behaviours. This booklet is adapted from previously developed materials used in the Physical Activity and Nutrition for Seniors project ${ }^{37}$ and modified to ensure suitability for the Vietnamese cultural context. 
Table 1 Education sessions linked to the theoretical basis of the study

\begin{tabular}{|c|c|c|}
\hline Themes & Contents & $\begin{array}{l}\text { Link with behaviour } \\
\text { change techniques }\end{array}$ \\
\hline $\begin{array}{l}\text { Session } 1 \text { (month } 1 \text { ): } \\
\text { introduction to the project }\end{array}$ & $\begin{array}{l}\text { 1. Getting to know each other } \\
\text { 2. Introduce the programme } \\
\text { 3. Benefits of physical activity and nutrition on health, } \\
\text { especially people with MetS, CVD, T2D } \\
\text { 4. Introduce and distribute the booklet } \\
\text { 5. Establishment of goal setting }\end{array}$ & $\begin{array}{l}\text { Interaction with other } \\
\text { participants; } \\
\text { Outcome expectation }\end{array}$ \\
\hline $\begin{array}{l}\text { Session } 2 \text { (month 2): physical } \\
\text { activity }\end{array}$ & $\begin{array}{l}\text { 1. Demonstrate and practice of exercises and walking, } \\
\text { programme such as warm up, cool down } \\
\text { 2. Barriers and enablers of being physically active } \\
\text { 3. How to identify and overcome these barriers }\end{array}$ & $\begin{array}{l}\text { Observational learning; } \\
\text { Self-efficacy; } \\
\text { Outcome expectation }\end{array}$ \\
\hline $\begin{array}{l}\text { Session } 3 \text { (month 3): healthy } \\
\text { diet }\end{array}$ & $\begin{array}{l}\text { 1. Demonstration and discussion of healthy foods and } \\
\text { healthy eating habits } \\
\text { 2. Barriers and enablers of maintaining a healthy diet } \\
\text { 3. How to identify and overcome barriers }\end{array}$ & $\begin{array}{l}\text { Observational learning; } \\
\text { Self-efficacy; } \\
\text { Outcome expectation }\end{array}$ \\
\hline $\begin{array}{l}\text { Session } 4 \text { (month } 4 \text { ): } \\
\text { experience and lessons learnt }\end{array}$ & $\begin{array}{l}\text { 1. Interactive session on overcoming barriers to being } \\
\text { physically active and maintaining a healthy diet } \\
\text { 2. Discussion of food types } \\
\text { 3. Feedback from participants } \\
\text { 4. Encourage participants to read the relevant sections in the } \\
\text { booklet to reinforce what they learnt in the sessions } \\
\text { 5. Review physical activity and nutrition goals }\end{array}$ & $\begin{array}{l}\text { Observational learning; } \\
\text { Reinforcement }\end{array}$ \\
\hline
\end{tabular}

The educational materials are simple, short and easy to understand and comprise four sections. The first section introduces the programme as well as the benefits of physical activity and a healthy diet. The second section focuses on various modes of physical activity, and contains illustrations and tips on how to perform physical activity (eg, brisk walking) correctly and resistance, weight training and stretching exercises safely. The third section contains suggested meal plans, recipes and tips for healthy eating, encouraging a higher consumption of fruits, vegetables and fibre while reducing intake of saturated fat, salt and sugar. The last section provides information on the barriers to physical activity and healthy eating and how to overcome them, together with practical examples. Participants will also be asked to set their own physical activity and nutrition goals and to enter their progress and achievements towards these goals in the booklet. Figure 3 shows the front page of the information booklet (available from the first author on request).

\section{Resistance exercises}

Each participant will be provided with a resistance band. Detailed instructions on usage, warming up and exercise techniques, as well as how to develop their own personal physical activity goals and plan, will be disseminated during the education sessions. Written information and pictorial illustrations are presented in the booklet.

\section{Walking groups}

Four walking groups per commune will be established immediately after the first education session. These walking groups serve to mobilise the participants, supporting them to meet and walk together in order to achieve the physical activity guidelines for health benefits. All participants will be encouraged to join a walking group based on their geographic location within the commune and guided by their local walk leaders.

\section{Outcome measures}

Table 2 summarises the outcome variables and their corresponding measuring instruments.

\section{Blood samples}

Blood samples will be collected by a phlebotomist in the morning after fasting for at least 8 hours during the community clinic visit at baseline and at 6-month post-test. The collected blood samples will be stored at $2-8^{\circ} \mathrm{C}$ in iceboxes and then transported to the Hanam provincial biochemistry laboratory for analysis within 6 hours. Plasma glucose will be measured by the glucose oxidase method. Lipid profile including total cholesterol, triglycerides and HDL-C will be measured by enzymatic methods. Glucose and lipids will be analysed using a semiautoanalyser (Screen Master Laboratory; Hospitex Diagnostics LIHD112, Italy) with a commercial kit (Chema Diagnostica, Italy).

\section{Anthropometric measurements}

These will be taken by the programme staff at baseline and at 6-month post-test following the WHO's guidelines. ${ }^{38}$ Such measurements include height, weight, waist and hip circumferences using a portable stadiometer, tape measures, and calibrated electronic scales. 


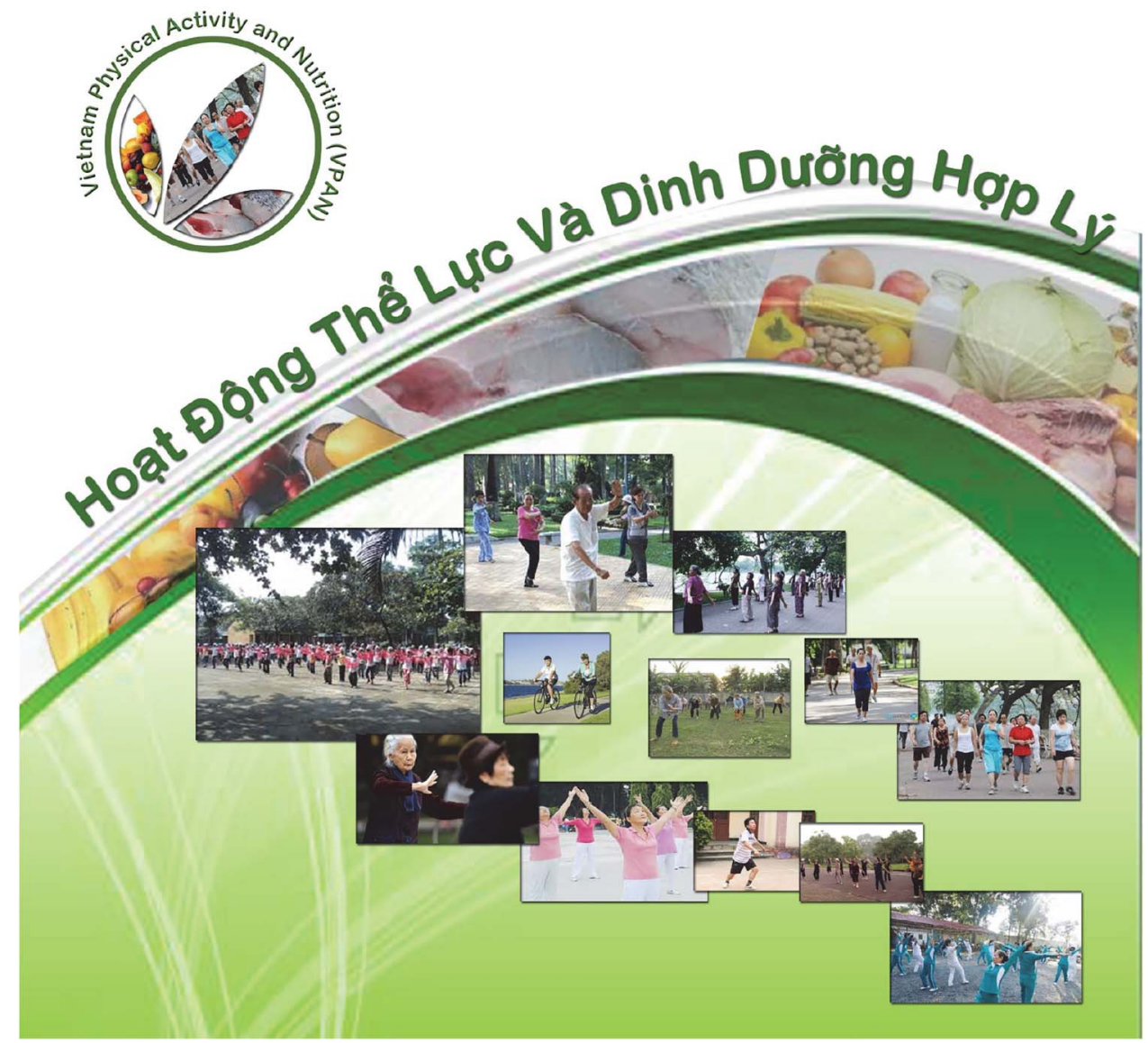

Figure 3 Front page of the information booklet.

\section{Blood pressure}

A physician or trained nurse will measure blood pressure using an Omron HEM-8712 automatic blood pressure monitor, with participants sitting and their arm supported at heart level. A mean value will be obtained after taking three consecutive measurements at baseline and post-test.

\section{Physical activity}

The validated International Physical Activity Questionnaire-Short Form (IPAQ-SF; Vietnamese version) ${ }^{39}$ will be administered to measure self-reported physical activity. Pedometers will also be used to objectively measure walking. The device will be fitted to the hip and worn for 7 consecutive days at baseline and post-test for the intervention group participants only.

\section{Diet}

The brief dietary habit questionnaire is adapted from the STEPS developed by the WHO. ${ }^{38}$ It will undergo pilot testing with further modifications before actual application to suit the local context. Information on cigarette smoking and alcohol drinking will also be solicited.

\section{Demographics}

Participant demographics include gender, age, education level, marital status and occupation. The structured questionnaire will be administered face to face.

\section{Process evaluation}

This type of evaluation assesses the following components of an intervention: fidelity (quality); dose delivered (completeness); dose received (exposure and satisfaction); reach (participation rate); recruitment and context (aspects of the environment influencing implementation or outcomes) ${ }^{40}$ It will be conducted midway through the intervention using a brief questionnaire, asking participants to evaluate the resources (ie, readability, how easy to understand, usefulness of advice, suitability and relevance to their age group). An open-ended section will enable participants to comment specifically on features they like/dislike. ${ }^{41}$ Process evaluation is important to identify the suitability of the intervention to the target group, and assesses the appropriateness of all aspects of the research procedure encompassing recruitment, data collection, evaluation, intervention implementation and dissemination. ${ }^{40}$

\section{Exit interview}

A total of 24 intervention participants will be randomly selected to participate in these interviews. The sample size is similar to that of a previous study. ${ }^{34}$ While the literature has identified that sample size recommendations for non-probabilistic, purposive qualitative studies can range from 5 to 25 participants, saturation occurs within the first 12 interviews. ${ }^{42}$ Therefore, 12 programme 
Table 2 Summary of outcome measures

\begin{tabular}{|c|c|}
\hline Outcome variables & Measuring instrument \\
\hline \multicolumn{2}{|l|}{ Primary outcomes } \\
\hline $\begin{array}{l}\text { Physical activity: walking, intensity, duration and } \\
\text { frequency }\end{array}$ & IPAQ-SF, pedometer \\
\hline $\begin{array}{l}\text { Diet: fat, oil, salt, sugar intake, fruit and vegetable } \\
\text { consumption }\end{array}$ & $\begin{array}{l}\text { Modified dietary behaviour questionnaire from the } \\
\text { WHO STEPS survey }\end{array}$ \\
\hline \multicolumn{2}{|l|}{ Secondary outcomes } \\
\hline Plasma glucose, triglyceride, total cholesterol and HDL-C & Fasting blood sample \\
\hline $\begin{array}{l}\text { Anthropometric measurements: height, weight, waist } \\
\text { circumference, hip circumference, blood pressure }\end{array}$ & $\begin{array}{l}\text { Portable stadiometer, tape measure, calibrated electronic } \\
\text { scales, Omron HEM-8712 automatic blood pressure monitor }\end{array}$ \\
\hline
\end{tabular}

completers and 12 non-completers will be asked if they are willing to participate in a face-to-face interview to gain information regarding their perceptions of the intervention programme and resources. The noncompleters will be asked reasons for their withdrawal. It is estimated that each interview will take $<20 \mathrm{~min}$. Permission will be sought for recording the interviews.

\section{Statistical analysis}

In addition to descriptive statistics, generalised mixed regression models will be fitted to the repeated pre-post measures to evaluate the magnitude of the changes and associated effect sizes. Effectiveness of the proposed intervention, and comparisons between the intervention group and the control group over time, will be ascertained via the group by time interaction term in the multivariate regression models, taking into account the repeated measures, correlated data structure and the presence of missing observations, whereas intention-to-treat analysis will be undertaken to assess sensitivity of the inferences. All data entry and statistical analyses will be performed in the STATA package Release V.13.

\section{Ethics and dissemination}

All participants will be provided with an information sheet (see online supplementary file 1) and asked by the trained programme staff to sign a consent form (see online supplementary file 2) prior to entry into the programme and be made aware that they are free to withdraw at any time. Only aggregated data will be reported in publications. The research protocol was approved by the Curtin University Human Research Ethics Committee (approval number: HR139/2014). The results of the study will be disseminated through publications, reports and conference presentations.

\section{DISCUSSION}

Vietnamese adults aged 50-65 years account for $13.8 \%$ (equivalent to 12.4 million people) of the total population, ${ }^{43}$ while $40 \%$ of the middle-aged population have pre-MetS. ${ }^{16}$ Therefore, this study will contribute to the knowledge base of the risk factors associated with MetS and related chronic diseases in Vietnam. It is the first community-based RCT ever undertaken on Vietnamese adults with MetS according to our extensive literature search. Findings from the study will be potentially applicable for NCD prevention in Vietnam.

Contributors VDT coordinated the programme and drafted the manuscript. VDT, AHL, JJ, APJ, PH and LTPM designed the study and revised the manuscript. All authors have read and approved the final version for publication.

Funding This study is financially supported by the School of Public Health, Curtin University, Perth, Western Australia, Australia.

Competing interests None declared.

Patient consent Obtained.

Ethics approval Curtin University Human Research Ethics Committee (approval number: HR139/2014).

Provenance and peer review Not commissioned; externally peer reviewed.

Open Access This is an Open Access article distributed in accordance with the Creative Commons Attribution Non Commercial (CC BY-NC 4.0) license, which permits others to distribute, remix, adapt, build upon this work noncommercially, and license their derivative works on different terms, provided the original work is properly cited and the use is non-commercial. See: http:// creativecommons.org/licenses/by-nc/4.0/

\section{REFERENCES}

1. General Statistics Office of Vietnam. Area, population and population density by province, Hanoi 2015. http://www.gso.gov.vn/default_en. aspx?tabid=774 (accessed 9 Jan 2016).

2. Hoy D, Rao C, Nhung NTT, et al. Risk factors for chronic disease in Viet Nam: a review of the literature. Prev Chronic Dis 2013;10:120067.

3. Harper C. Vietnam noncommunicable disease prevention and control programme 2002-2010: implementation review. WHO Western Pacific Region, 2011.

4. Alberti KG, Eckel RH, Grundy SM, et al. Harmonizing the metabolic syndrome: a joint interim statement of the International Diabetes Federation Task Force on Epidemiology and Prevention; National Heart, Lung, and Blood Institute; American Heart Association; World Heart Federation; International Atherosclerosis Society; and International Association for the Study of Obesity. Circulation 2009;120:1640-5.

5. International Diabetes Federation. The IDF consensus worldwide definition of the metabolic syndrome. Belgium: IDF, 2006.

6. Kaur J. A comprehensive review on metabolic syndrome. Cardiol Res Pract 2014;2014:943162.

7. WHO. Definition diagnosis and classification of diabetes mellitus and its complications. Report of a WHO consultation. Part 1: diagnosis and classification of diabetes mellitus. Geneva: WHO, 1999.

8. National Cholesterol Education Program (NCEP) Expert Panel on Detection, Evaluation, and Treatment of High Blood Cholesterol in Adults (Adult Treatment Panel III). Third report of the National Cholesterol Education Program (NCEP) expert panel on detection, 
evaluation, and treatment of high blood cholesterol in adults (Adult Treatment Panel III) final report. Circulation 2002;106:3143-421.

9. Blaha MJ, Bansal S, Rouf R, et al. A practical "ABCDE" approach to the metabolic syndrome. Mayo Clin Proc 2008;83:932-41.

10. Grundy SM, Cleeman JI, Daniels SR, et al. Diagnosis and management of the metabolic syndrome: an American Heart Association/National Heart, Lung, and Blood Institute Scientific Statement. Circulation 2005;112:2735-52.

11. Kolovou GD, Anagnostopoulou KK, Salpea KD, et al. The prevalence of metabolic syndrome in various populations. Am J Med Sci 2007;333:362-71.

12. Son le NT, Kunii D, Hung NT, et al. The metabolic syndrome: prevalence and risk factors in the urban population of Ho Chi Minh City. Diabetes Res Clin Pract 2005;67:243-50.

13. Trinh OT, Nguyen ND, Phongsavon $P$, et al. Metabolic risk profiles and associated risk factors among Vietnamese adults in $\mathrm{Ho}$ Chi Minh City. Metab Syndr Relat Disord 2010;8:69-78.

14. Hong TK, Trang NH, Dibley MJ. Prevalence of metabolic syndrome and factor analysis of cardiovascular risk clustering among adolescents in Ho Chi Minh City, Vietnam. Prev Med 2012:55:409-11.

15. Duc Son LN, Kusama K, Hung NT, et al. Prevalence and risk factors for diabetes in Ho Chi Minh City, Vietnam. Diabet Med 2004;21:371-6.

16. Binh TQ, Phuong PT, Nhung BT, et al. Metabolic syndrome among a middle-aged population in the Red River Delta region of Vietnam. BMC Endocr Disord 2014;14:77.

17. National Heart, Lung, and Blood Institute. Clinical guidelines on the identification, evaluation, and treatment of overweight and obesity in adults-the evidence report. National Institutes of Health. Obes Res 1998:6(Suppl 2):51s-209s.

18. Grundy SM, Cleeman JI, Daniels SR, et al. Diagnosis and management of the metabolic syndrome: an American Heart Association/National Heart, Lung, and Blood Institute scientific statement: executive summary. Crit Pathw Cardiol 2005;4:198-203.

19. Ilanne-Parikka P, Eriksson JG, Lindström J, et al. Effect of lifestyle intervention on the occurrence of metabolic syndrome and its components in the Finnish Diabetes Prevention Study. Diabetes Care 2008;31:805-7.

20. Burke L, Lee $\mathrm{AH}$, Jancey J, et al. Physical activity and nutrition behavioural outcomes of a home-based intervention program for seniors: a randomized controlled trial. Int J Behav Nutr Phys Act 2013;10:14

21. Burke L, Lee A, Pasalich M, et al. Effects of a physical activity and nutrition program for seniors on body mass index and waist-to-hip ratio: a randomised controlled trial. Prev Med 2012;54:397-401.

22. Yamaoka K, Tango T. Effects of lifestyle modification on metabolic syndrome: a systematic review and meta-analysis. BMC Med 2012;10:138.

23. Saneei P, Salehi-Abargouei A, Esmaillzadeh A, et al. Influence of Dietary Approaches to Stop Hypertension (DASH) diet on blood pressure: a systematic review and meta-analysis on randomized controlled trials. Nutr Metab Cardiovasc Dis 2014;24:1253-61.

24. Salehi-Abargouei A, Maghsoudi Z, Shirani F, et al. Effects of Dietary Approaches to Stop Hypertension (DASH)-style diet on fatal or nonfatal cardiovascular diseases-Incidence: a systematic review and meta-analysis on observational prospective studies. Nutrition 2013;29:611-18.
25. The Diabetes Prevention Program Research Group. The Diabetes Prevention Program (DPP): description of lifestyle intervention. Diabetes Care 2002;25:2165-71.

26. Folta SC, Lichtenstein AH, Seguin RA, et al. The Strong WomenHealthy Hearts Program: reducing cardiovascular disease risk factors in rural sedentary, overweight, and obese midlife and older women. Am J Public Health 2009:99:1271-7.

27. Holt AM, Jancey J, Lee AH, et al. A cluster-randomised controlled trial of a physical activity and nutrition programme in retirement villages: a study protocol. BMJ Open 2014;4:e005107.

28. Blackford K, Jancey J, Lee AH, et al. A randomised controlled trial of a physical activity and nutrition program targeting middle-aged adults at risk of metabolic syndrome in a disadvantaged rural community. BMC Public Health 2015;15:284.

29. WHO Expert Consultation. Appropriate body-mass index for Asian populations and its implications for policy and intervention strategies. Lancet 2004;363:157-63.

30. Tran C. Assessment of the prevalence of obesity and related risk factors in Vietnamese adults living in urban areas of Ho Chi Minh City, Vietnam. Australia: University of Newcastle, 2004.

31. Bandura A. Self-efficacy: the exercise of control. New York: Freeman, 1997.

32. Glanz K, Rimer B, Viswanath K. Health behaviour and health education: theory, research and practice. 4th edn. Sanfrancisco: John wiley \& Sons, 2008.

33. Artinian NT, Fletcher GF, Mozaffarian D, et al. Interventions to promote physical activity and dietary lifestyle changes for cardiovascular risk factor reduction in adults: a scientific statement from the American Heart Association. Circulation 2010;122:406-41.

34. Burke L, Jancey J, Howat PA, et al. Physical activity and nutrition program for seniors (PANS): protocol of a randomized controlled trial. BMC Public Health 2010;10:751.

35. Lee EG, Choi JH, Kim KE, et al. Effects of a walking program on self-management and risk factors of metabolic syndrome in older Korean adults. J Phys Ther Sci 2014;26:105-9.

36. Hollis JF, Gullion CM, Stevens VJ, et al. Weight loss during the intensive intervention phase of the weight-loss maintenance trial. Am J Prev Med 2008;35:118-26.

37. Burke L, Howat P, Lee A, et al. Development of a nutrition and physical activity booklet to engage seniors. BMC Res Notes 2008;1:1-7.

38. World Health Organisation. WHO STEPS surveillance manual. Geneve, 2008.

39. Tran DV, Lee AH, Au TB, et al. Reliability and validity of the International Physical Activity Questionnaire-Short Form for older adults in Vietnam. Health Promot J Austr 2013;24:126-31.

40. Nutbeam D. Evaluation in a nutshell: a practical guide to the evaluation of health promotion programs. North Ryde, NSW: McGraw-Hill, 2006.

41. Burke L, Jancey JM, Howat $P$, et al. Physical activity and nutrition program for seniors (PANS): process evaluation. Health Promot Pract 2012;14:543-51.

42. Guest G, Bunce A, Johnson L. How many interviews are enough? An experiment with data saturation and variability. Field Methods 2006;18:59-82.

43. General Statistics Office of Vietnam of Vietnam. The 1/4/2012 time-point population change and family planning survey: major findings. Hanoi: General Statistics Office, 2012. 